OCEANS 2016 MTS/IEEE. 19-23 Sept. 2016, Monterey, CA, USA.

Pages 1-9

http://dx.doi.org/10.1109/OCEANS.2016.7761460

http://archimer.ifremer.fr/doc/00383/49392/

(C) 2016 IEEE

\title{
Application of Hilbert-Huang decomposition to temperature and currents data in the Réunion island
}

\author{
Kbaier-Ben Ismail Dhouha ${ }^{1,{ }^{*},}$, Lazure Pascal ${ }^{2}$, Puillat Felix Ingrid ${ }^{2}$
}

1 IUniversity of Bedfordshire, Department of Computer Science and Technology, Luton, UK

${ }^{2}$ IFREMER, ODE/DYNECO/PHYSED, France

* Corresponding author :Dhouha Kbaier-Ben Ismail, email address : dhouha.kbaier@telecombretagne.eu

\begin{abstract}
:
In marine sciences, time series are often nonlinear and nonstationary. Their analysis faces new challenges and thus requires the implementation of adequate and specific methods. We use the HilbertHuang Transform (HHT) for the spectral analysis of high frequency sampled time series in near shore waters of the Réunion island, located in the Indian Ocean $700 \mathrm{~km}$ east of Madagascar. We focus particularly on automatic measurements of sea level fluctuations, temperature records and current data sets at four different stations in the island. We look at the contribution of different Intrinsic Mode Functions (IMFs) obtained by the Empirical Mode Decomposition (EMD) and also compare the Hilbert spectra with the wavelet spectra. The inertial wave and several low-frequency tidal waves are identified by the application of EMD. Furthermore, the authors investigate the cross-correlations between data at the different stations. Wavelet coherence and EMD based Time Dependent Intrinsic Correlation (TDIC) analyses are applied to consider the correlation between two nonstationary time series. By TDIC analysis, it was concluded that the high frequency modes have small correlation; whereas the trends are perfectly correlated. The results obtained by wavelet coherence are very similar, thus confirming that both approaches could be used for identification of main properties of marine environmental time series. The methodologies presented in this paper are general and thus can be applied on other time series from the environmental and oceanic sciences, where time series are complex with fluctuations over a large range of different spatial and temporal scales, from seconds to thousands of years.
\end{abstract}

\section{Introduction}

Surface tides are considered the heartbeat of our planet's oceans. They are deterministic since they are the result of the gravitational pull of the moon and sun on the earth, as well as the rotation of the earth itself. Moreover, internal tides are ubiquitous in stratified ocean. They result from the interaction

of the barotropic tides with the bottom topography of the oceans. By definition, internal tides involve vertical shear and thus lead to mixing in the deep ocean. They play a role in dissipating tidal energy. They can propagate over great distances of hundreds of $\mathrm{km}$ and distort the density profile due to their large amplitude. In situ time series of temperature and currents are then necessary to understand their properties and how they propagate and modify their shape to the coast. 
Moreover, temperature is one of the important environmental factors. For instance, it is an important factor to consider when assessing water quality since it is highly correlated with oxygen concentration. In this paper, internal tides in the Réunion island are investigated by temperature and bottom pressure observations at four coastal stations during the period from July 2011 to January 2012.

Although Fourier-based spectral analysis methods have been widely used for studying time series, they face constraining limitations such as nonlinearity of the processes. The assumption of linear superposition of wave components spreads the energy of a nonlinear wave into many harmonics, which are phase-coupled via the nonlinear dynamics inherent in ocean waves. In addition to the nonlinearity issue, Fourier spectral analysis should be used for periodic and stationary processes only.

In marine environment, the recorded time series are often nonlinear, nonstationary and interact with each other (Cazelles et al., 2008; Ghorbani, 2013; Rao and Hsu, 2008). Thus, standard Fourier spectral analysis is inappropriate. Continuous Wavelet Transform (CWT) is one of the most commonly used techniques for the time-frequency analysis of time series (Torrence and Compo, 1998). On the other hand, the introduction of Empirical Mode Decomposition (EMD) by Huang et al. $(1998,1999)$ represents a fundamentally different approach for decomposing nonlinear and nonstationary signals. The associated spectral analysis, namely the Hilbert-Huang Transform (HHT), is a popular approach used today for analyzing such time series. It has already been applied in marine sciences (Huang and $\mathrm{Wu}, 2008$ ). For example, Dätig and Schlurmann (2004) applied HHT to show excellent correspondence between simulated and recorded nonlinear waves. Schmitt et al. (2009) applied the HHT method to characterize the scale invariance of velocity fluctuations in the surf zone. The EMD scheme was used in studying sea level rise (Ezer et al., 2013). Yin et al. (2014) also applied the method and identified three kinds of low-frequency waves using some observations in the coastal water of the East China Sea.

In this paper, sea level fluctuations, temperature records and currents time series obtained from automatic measurements in near shore waters of the Réunion island are considered. These high frequency measurements are recorded every ten minutes from July 2011 to January 2012. The two time-frequency decomposition methods are applied and 
discussed hereafter to study the existence and propagation of internal waves with objective to analyze the spectral content of such a complex signal. The paper is structured in six sections. After the description of the background and objectives in section I, the time series used for practical analyses are introduced in section II. CWT and HHT approaches are presented in section III. Section IV deals with both EMD based and wavelet based cross correlation methods: Time Dependent Intrinsic Correlation (TDIC) and wavelet coherence. Furthermore, the authors discuss in section V how the HHT differs from other time-frequency analysis tools with respect to the spectral frequencies and smearing challenges. Finally, section VI brings out the conclusions of this paper.

\section{PResentation OF THE EXPERIMENTAL DAtABASE}

Automatic measurements in $40 \mathrm{~m}$ depth waters of the Réunion island are considered. Using Acoustic Doppler Current Profilers (ADCP), bottom temperatures, currents and sea level fluctuations are recorded every ten minutes from $21^{\text {st }}$ July 2011 to $19^{\text {th }}$ January 2012 in four different stations $\left(S_{1}\right.$, $\mathrm{S}_{2}, \mathrm{~S}_{3}$ and $\mathrm{S}_{4}$ ) shown in Fig. 1. Hereafter, we denote the temperature time series by Temp 1, Temp 2, Temp 3 and Temp 4 . For the sea level, the time series are denoted by $s l_{1}, s l_{2}, s l_{3}$ and $s l_{4}$ respectively. The authors consider also the four currents time series recorded at $20 \mathrm{~km}$ distance. The along shore currents are denoted by $U_{1}, U_{2}, U_{3}$ and $U_{4}$; whereas the cross shore currents are denoted by $V_{1}, V_{2}, V_{3}$ and $V_{4}$. These time series, plotted in Fig. 2 and Fig. 3, are nonstationary. In the next section, we perform adequate power spectral analysis of these data.

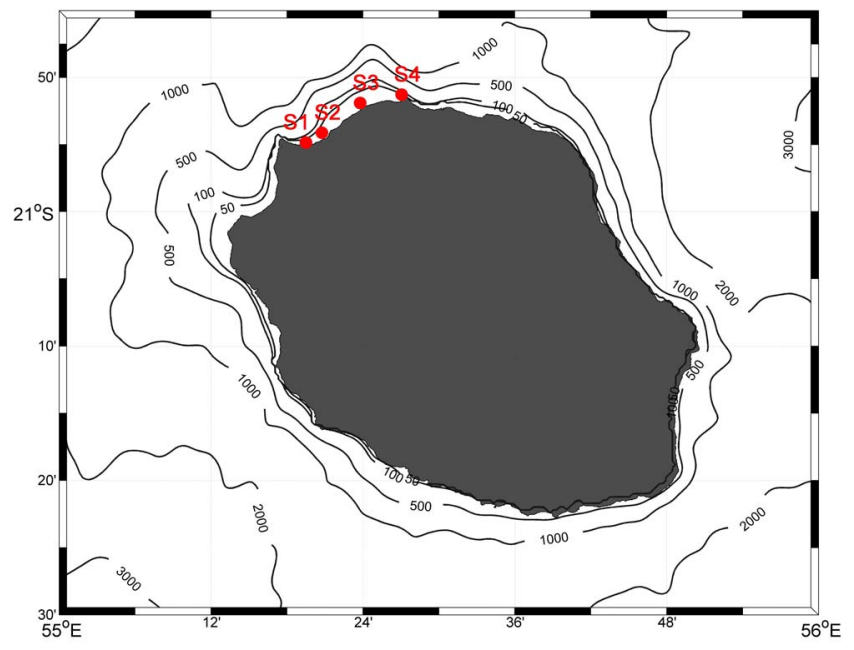

Fig. 1. Locations and bathymetry of the four measurement stations.

\section{HILBERT-HUANG TRANSFORM}

In Earth sciences, time series are often unevenly spaced and nonstationary. Thus, standard Fourier spectral analysis is inappropriate. To accommodate the variety of data generated by nonlinear and nonstationary processes, Huang et al. (1998) developed a new adaptive data analysis method designated by the NASA as the HHT and introduced hereafter. The HHT consists of two steps: EMD and Hilbert Spectral Analysis
(HSA). In the next subsections, we explain how HHT offers a potentially viable method for nonlinear and nonstationary data analysis, especially for time-frequency-energy representations.
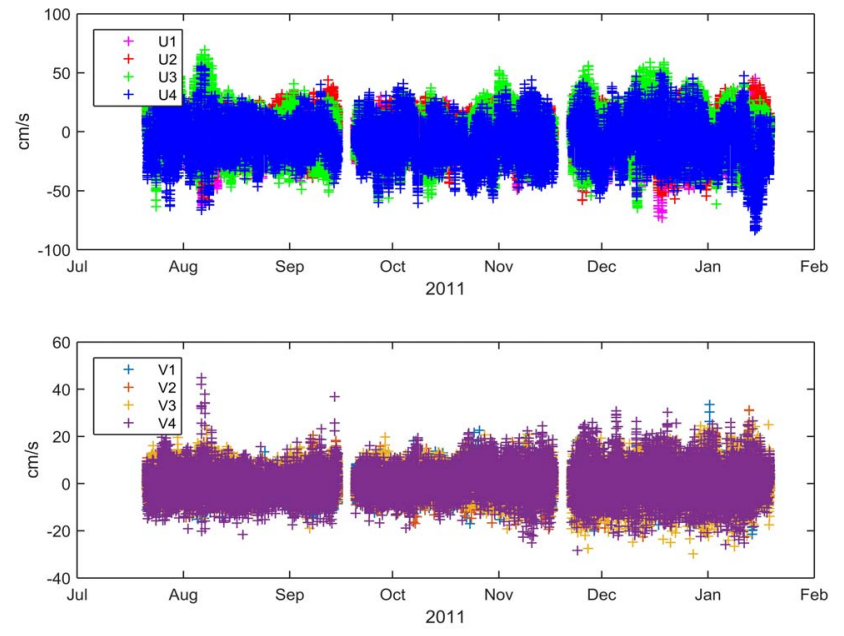

Fig. 2. Along shore and cross shore currents recorded in the Réunion at four stations. Note the two-week missing data in mid-September and by the end of November 2011.
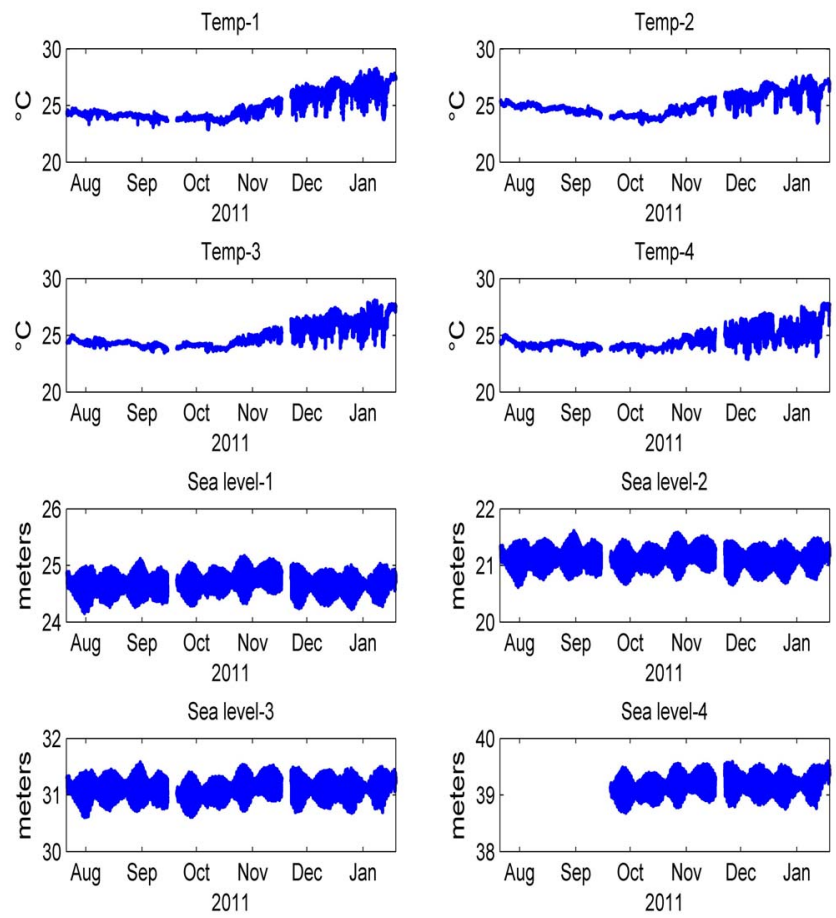

Fig. 3. Bottom temperatures and sea level fluctuations recorded at four stations in the Réunion island.

\section{A. Empirical mode decomposition}

The key part of HHT is EMD which has been tested and validated exhaustively (Huang and Attoh-Okine, 2005; Huang and Shen, 2005), though only empirically. With EMD, any 
complicated data set can be decomposed into a finite and often small number of Intrinsic Mode Functions (IMFs). An IMF is defined as a function having the same number of extrema and zero-crossings. It has also symmetric upper and lower envelopes defined by the local maxima and minima respectively. The IMFs represent different scales of the original time series and physically meaningful modes. Indeed, the EMD divides the time series into a series of modes, but unlike a Fourier transform where each component has a constant frequency and a cosine or sine shape, the EMD allows each mode to have a time-dependent frequency and amplitude. In contrast to almost all the previous methods, the EMD works directly in temporal space rather than in the corresponding frequency space. With the definition of the IMFs, one can then decompose any function $x(t)$ through a sifting process:

- $\quad$ First identify all the local extrema and then connect all the local maxima (minima) by a cubic spline line to form the upper (lower) envelope. The envelopes should encompass all the data between them.

- $\quad$ Calculate $m_{l}$, the running mean of the envelope.

- Treat the difference $\mathrm{x}(\mathrm{t})=m_{1}-h_{1}$ as the data in the next iteration and repeat the above sifting processing until $h_{l}$ becomes an IMF.

○ $h_{1}-m_{1(1)}=h_{1(1)}$.

- After $k$ times, the first IMF is derived: $h_{1(k-1)}-m_{1(k)}=h_{1(k)}=c_{1}$. The term $c_{1}$ contains the highest frequency component of the original signal.

- Separate $c_{1}$ from the rest of the data: $r_{1}=x(t)-c_{1}$ and carry out the same process described above.

- Repeat the procedure until $r_{n}$ becomes a constant, a monotonic function or a function with only one maximum and one minimum from which no more IMF can be extracted:

$$
\begin{array}{ll}
\circ & r_{1}-c_{2}=r_{2} \\
\circ & \cdots \\
\circ & r_{n-1}-c_{n}=r_{n}
\end{array}
$$

- By summing all the equations, we can get a decomposition with $n$ IMFs and a residue as follows:

$$
x(t)=\sum_{j=1}^{n} c_{j}(t)+r_{n}(t) \text {. }
$$

Due to a dyadic filter bank property of the EMD algorithm (Flandrin et al., 2004; Huang et al., 2008; Wu and Huang, 2004), usually in practice, the number of IMFs modes is less than $\log _{2}(N)$, where $N$ is the length of the data set. Unlike Fourier based methodologies and wavelet transform, the EMD method does not define a basis a priori (Flandrin and Gonçalvès, 2004; Huang et al., 1998, 1999) and the IMFs constitute an adaptive basis. Therefore, EMD is very suitable for nonstationary and nonlinear time series analysis (Huang et al., 1998, 1999). One way to express the nonstationarity is to find instantaneous frequency and instantaneous amplitude. This was the reason why Hilbert spectrum analysis was included as a part of HHT (Huang, 2005, Huang et al., 2011). It has been applied successfully in various research fields to characterize the energy-time-frequency in different topics (Abarca-Del-Rio and Mestre, 2006; Chen et al., 2010; Janosi and Müller, 2005; Martini et al., 2014; Schmitt et al., 2009; Tatli et al., 2005; Wu et al., 2006), to cite few. In the next subsection, we present HHT results for the currents recorded in the Réunion.

\section{B. HHT analysis results}

To analyze the variability, the HHT has been applied to the records at the four considered sites. For instance, the sea levels are divided into a series of modes using the EMD process. After EMD decomposition of $s l_{l}$, twelve IMFs modes are obtained plus the residual, as shown in Fig. 4. The time scale is increasing with the number of the IMF mode, thus the first IMF corresponds to the highest frequency.
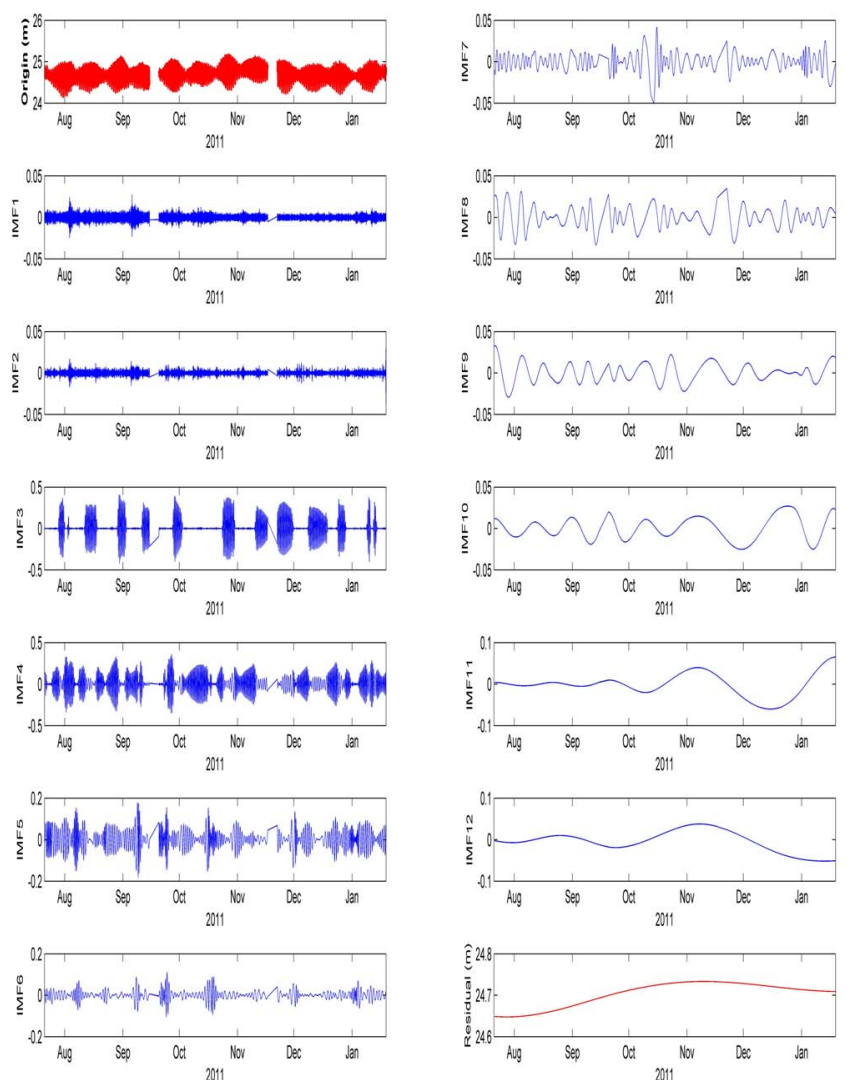

Fig. 4. The IMFs modes and the trend from EMD for sea level data set $s l_{l}$.

Table I shows several tidal constituents that are identified through the IMFs: diurnal (such as $K_{1}$ ), semidiurnal (such as the principal lunar semidiurnal constituent $M_{2}$. This constituent represents the rotation of the Earth with respect to the Moon), semimonthly waves (such as the Mf lunar constituent which expresses the effect of departure from a sinusoidal declinational motion), monthly waves (such as $\mathrm{Mm}$, the lunar monthly constituent which expresses the effect 
of irregularities in the Moon's rate of change of distance and speed in orbit). Moreover, the inertial period, defined as $\mathrm{T}_{\text {inertial }}=\frac{24}{2 \times \sin \varphi}($ Monin, 1990), is detected as an IMF mode ( see $I M F_{6}$ ). Table I gives also the contribution of each IMF to the total energy (measured by the variance), percentage of total and cumulative percentage contribution to the sum of the variances. Note that the most energetic IMFs ( $I M F_{3}$ and $I M F_{5}$ ) account for over $83 \%$ of the total energy.

TABLE I. THE MEAN PERIOD OF THE IMFS MODES FOR SEA LEVEL DATASET, THE IDENTIFIED TIDAL CONSTITUENTS, AND THE CONTRIBUTION OF EACH IMF TO THE TOTAL ENERGY.

\begin{tabular}{|c|c|c|c|c|c|}
\hline IMF & $\begin{array}{l}\text { Mean } \\
\text { Period } \\
\text { (days) } \\
\end{array}$ & $\begin{array}{l}\text { Identified } \\
\text { tidal wave }\end{array}$ & Variance & $\begin{array}{l}\% \text { of } \\
\text { total }\end{array}$ & $\begin{array}{c}\text { Cumul } \\
\text { ative }\end{array}$ \\
\hline$I M F_{1}$ & 0.02 & - & $1.35 \mathrm{e}-05$ & 0.04 & 0.04 \\
\hline$I M F_{2}$ & 0.05 & - & $6.68 \mathrm{e}-06$ & 0.02 & 0.07 \\
\hline$I M F_{3}$ & $\begin{array}{c}0.54 \text { days } \\
=12.88 \\
\text { hours }\end{array}$ & \multirow{2}{*}{$\begin{array}{l}\text { Semidiurnal } \\
M_{2}: 12.42 \mathrm{~h} \\
N_{2}: 12.66 \mathrm{~h} \\
K_{2}: 11.97 \mathrm{~h}\end{array}$} & 0.01 & $\begin{array}{c}44.0 \\
2\end{array}$ & \multirow{2}{*}{44.11} \\
\hline$I M F_{4}$ & $\begin{array}{c}0.57 \text { days } \\
=13.68 \\
\text { hours }\end{array}$ & & 0.01 & $\begin{array}{c}38.8 \\
8\end{array}$ & \\
\hline$I M F_{5}$ & $\begin{array}{c}0.99 \text { days } \\
=23.69 \\
\text { hours }\end{array}$ & $\begin{array}{c}\text { Diurnal } \\
K_{1}: 23.93 \mathrm{~h}\end{array}$ & $1.00 \mathrm{e}-03$ & 6.16 & 83.02 \\
\hline$I M F_{6}$ & $\begin{array}{c}1.38 \text { days } \\
=33.18 \\
\text { hours }\end{array}$ & $\begin{array}{l}\text { Inertial wave } \\
\begin{array}{c}2 \times \sin \left(21^{\circ}\right) \\
=33,485 \mathrm{~h}\end{array}\end{array}$ & $5.10 \mathrm{e}-04$ & 1.69 & 89.19 \\
\hline$I M F_{7}$ & 3.80 & & $1.35 \mathrm{e}-04$ & 0.45 & 90.88 \\
\hline$I M F_{8}$ & 7.78 & Weekly cycle & $1.48 \mathrm{e}-04$ & 0.49 & 91.32 \\
\hline$I M F_{9}$ & 14.59 & $\begin{array}{c}\text { Semimonthly } \\
M f: 13.66 \\
\text { days (d) } \\
M s f: 14.76 \mathrm{~d}\end{array}$ & $1.82 \mathrm{e}-04$ & 0.60 & 91.82 \\
\hline$I M F_{10}$ & 30.11 & $\begin{array}{c}\text { Monthly } \\
M m: 27.55 \mathrm{~d}\end{array}$ & $1.77 \mathrm{e}-04$ & 0.59 & 92.42 \\
\hline$I M F_{11}$ & 43.10 & - & $9.69 e-04$ & 3.20 & 93.00 \\
\hline$I M F_{12}$ & 121.10 & $\begin{array}{c}\text { Season in the } \\
\text { Réunion } \\
\text { (nearly } 4 \\
\text { months) }\end{array}$ & $1.00 \mathrm{e}-03$ & 3.79 & 96.20 \\
\hline Res. & NA & NA & $1.35 \mathrm{e}-05$ & 0.04 & 100.00 \\
\hline
\end{tabular}

Apart from the tidal constituents identified in Table I, other tidal waves are found by the EMD decomposition of the temperature dataset such as third-degree diurnal $\left(M_{3}\right)$ and fourth-degree diurnal $\left(M_{4}\right)$. The EMD decomposition of the temperature time series reveals that for the high frequencies, the biggest contribution comes from four most energetic IMFs: the semidiurnal wave, followed by the diurnal wave, the fourth-degree diurnal and finally the third-degree diurnal. For example, for $\mathrm{Temp}_{2}$ these IMFs account for over $20 \%$ of the total energy. Thus in contrast to the sea level analysis, the main part of energy is not carried by the very high frequency components but rather by intermediate and low ones, especially the seasonal wave in accordance with the low frequency described by Pous et al., 2014.

Fig. 5 shows the estimated mean period $\bar{T}$ of each IMF mode in a semilog plot for Temp 1 and Temp 2 . The IMF modes of the two datasets have very nearly the same mean period and the changes in mean period from one mode to the means are a very nearly exponential function of the mode number $n$, i.e., $\bar{T}(n)=\alpha \times \gamma^{n}$ with $\alpha=0.037$ and $\gamma=1.84$ obtained by using a least square fitting algorithm. This value is close to 2 , which indicates a quasi-dyadic filter bank property of the EMD algorithm for these series, as found in other situations (Flandrin et al., 2004; Huang et al., 2008; Wu and Huang, 2004). In other words, there is an exponential increase with a slope very close to 2 since the mean scale of each mode series is 1.84 times the mean scale of the previous one. While the dyadic filter bank property allows to validating the EMD decomposition, it is fair to point out that this represents a drawback of the method since the EMD cannot distinguish frequencies that are close to each other.

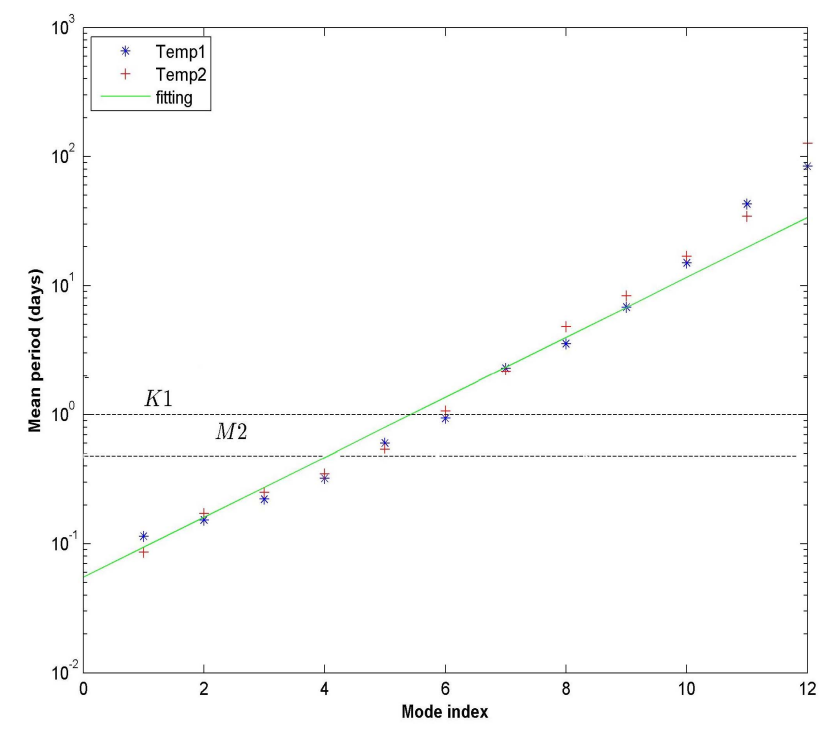

Fig. 5. Mean period of IMF modes for $T e m p_{1}$ and Temp $p_{2} K_{1}$ and $M_{2}$ are indicated by dashed lines.

\section{Wavelet and Hilbert spectra}

The CWT is a consecutive pass-band filter through time series, recognized as a very successful approach for the analysis of time series (Addison, 2010; Avdakovic et al., 2013; Torrence and Compo, 1998). The wavelet transform is widely used as a time-frequency analysis technique to deal with nonstationary signals. The choice of the mother wavelet is usually dependent on the type of data to deal with. HHT, on the other hand, does not require any convolution of the signal with a predefined basis function or mother wavelet. The process of decomposition is data-driven. Comparisons of wavelets with HHT are investigated in this subsection.

After EMD, the Hilbert-Huang spectrum allows the amplitude and the instantaneous frequency to be represented 
in a three-dimensional plot, in which the amplitude is the height in the time-frequency plane. This spectral representation offers a totally different interpretation when compared to the classical Fourier representation. In Fourier terms, the existence of energy at some frequency implies the existence of a component of a sine or a cosine wave throughout the whole time series or a significant part of it.

For sea level $s l_{l}$, the Hilbert spectrum is shown in Fig. 6, while the Morlet wavelet spectrum is shown in Fig. 7. Both the wavelet and the Hilbert spectrum show a similar timeenergy-frequency distribution, but the Hilbert spectrum is more quantitative. According to both the wavelet and the Hilbert spectra, the fluctuations of the frequency indicate that the tide is actually nonlinear at the measurement station. This is reasonable for all the tidal stations since the interaction of the tide with the near shore bathymetry made their profile nonlinear, even if the generating forces and the governing equations are linear. Moreover, sea level fluctuations are also influenced by meteorological effects and the mesoscale circulations which are mainly stochastic processes.

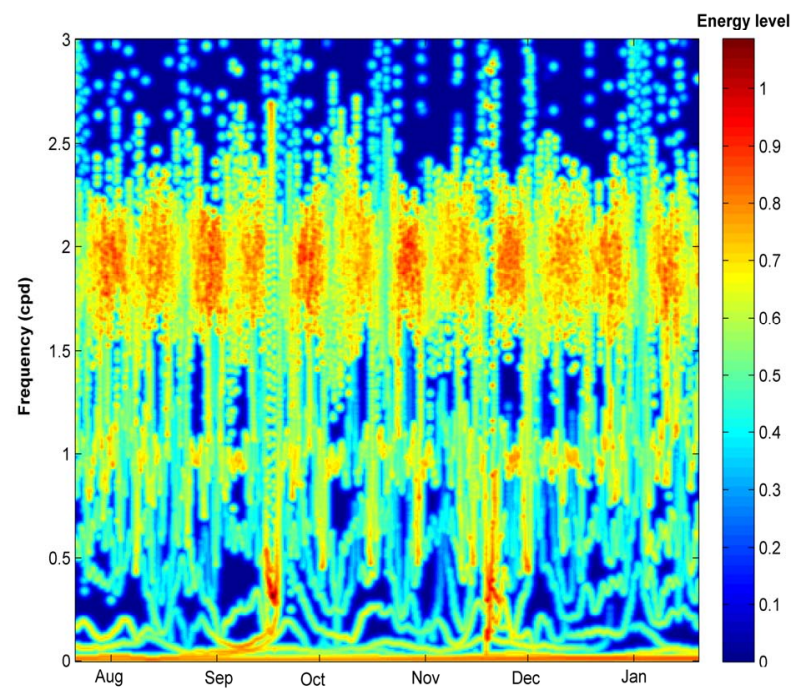

Fig. 6. Time-frequency analysis of $s l_{1}$ : Hilbert energy spectrum after EMD. Red/Blue colors indicate high/low variability.

In Fig. 6, the energy appears in skeleton lines representing each IMF, whereas the energy appears in smoothed contours for the wavelet spectrum in Fig. 7. The energy level in the Hilbert spectrum is given in a color scale. From this graph it is possible to see a detailed record of the variation in frequency and energy with time. For example, one can see that most of the energy is concentrated in a band around a frequency of 2 cycles per day $(c p d)$. Although there are low frequency components present in the spectrum, most of the energy is in the high frequencies, represented by IMFs 3, 4, 5 and 6 . Furthermore, the discontinuous, filamentary aspect of the Hilbert spectrum indicates a number of phase dropouts which shows that the data are nonstationary. According to both these spectra, the most energetic frequencies are the semidiurnal and the diurnal waves. For example, Fig. 7 shows horizontal clusters of peaks at frequencies of $1 \mathrm{cpd}$ and $2 \mathrm{cpd}$. It clearly shows significant periodicities at these cycles that are present through the entire time series $s l_{l}$. Nevertheless, the $1 \mathrm{cpd}$ is relatively weak (less significant) compared with the $2 \mathrm{cpd}$. The disadvantage of the wavelet power spectral analysis, however, is the requirement of evenly-spaced data. The effects of the linear interpolation can be seen in Fig. 7. Two long blue lines are observed in mid-September and mid-November. They correspond to the loss of the high frequency at these dates.

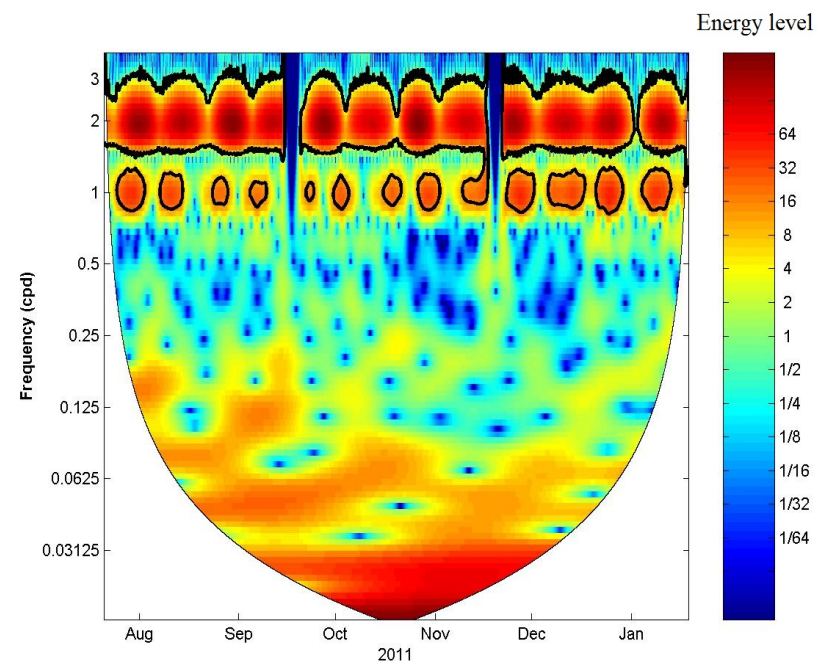

Fig. 7. The Morlet wavelet spectrum of the sea level data set $s l_{l}$. The two blue lines are due to the linear interpolation of the missing data at the corresponding dates.

Another example is the CWT of Temp 1 and Temp $_{2}$ provided by Fig. 8. We have superimposed the mean period of the IMFs obtained from Temp 1 and Temp 2 time series on the corresponding wavelet spectra. A noticeable similarity between the two methods is also observed although poor lowfrequency resolution is discerned for the Morlet wavelet spectra (no thick black contour beyond 1 cycle per day). For the CWT, a first wintry period is observed in July-October; followed by the summer beginning in the end of November and extending through the end of the record (the end of January), the period of high variability in the spectrum. This is consistent with the records of Fig. 3, since the maximum recorded temperatures are obtained in December-January. Indeed, during the Réunion summer, vertical stratification of the water column combined with horizontal oscillatory currents creates bottom temperature fluctuations, as observed in similar islands of the Pacific Ocean (Leichter et al., 2012). Such energetic fluctuations can be seen every few days. Further details on the Hilbert and wavelet spectra of the temperature data set are provided by Kbaier Ben Ismail et al., 2015.

As the signal content is now known in the frequency domain, the focus will shift to the application of an EMD based TDIC, in order to study the possible links between physical observations; and we compare TDIC with wavelet coherence. 

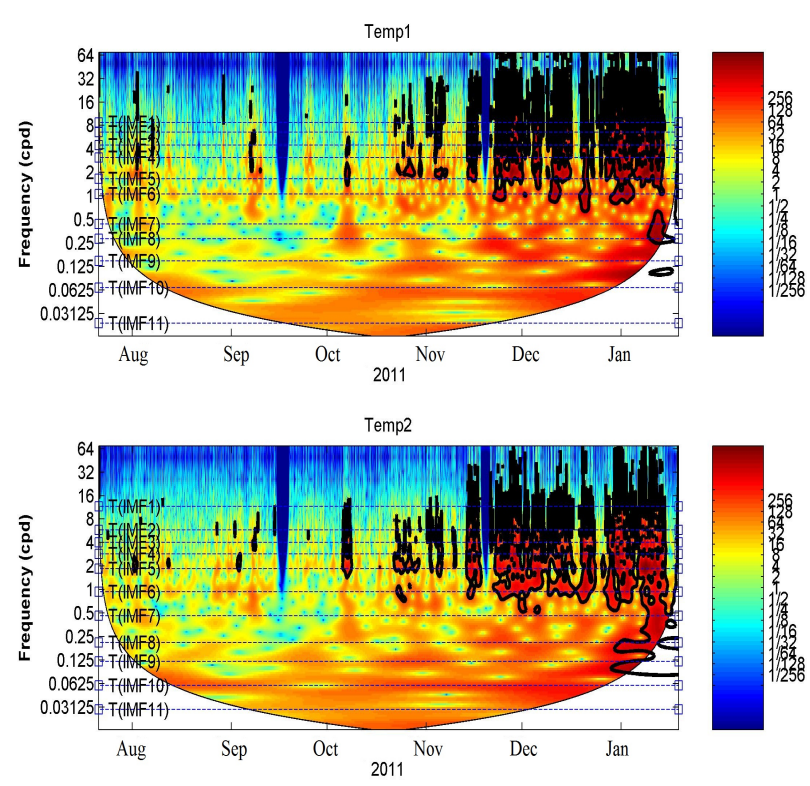

Fig. 8. Continuous wavelet power spectrum versus IMFs for Temp 1 and Temp 2 .

\section{EMD BASED AND WAVELET BASED CROSS CORRELATIONS}

The classical global expression for the correlation (defined as the covariance of two variables divided by the product of the standard deviation of the two variables) assumes that the variables should be stationary and linear. Applied to nonstationary time series, the cross correlation information may be altered and distorted. Many scientists tried to address the problem of nonsense correlations through different ways. In the following, we focus on both EMD based and wavelet based cross correlations.

\section{A. Time dependent intrinsic correlation}

In 2010, Chen et al. introduced an approach based on EMD. They proposed to first decompose the nonlinear and nonstationary data into their IMFs, then use the instantaneous periods of the IMFs to determine an adaptive window and finally compute the time dependent intrinsic correlation coefficients. Huang and Schmitt (2014) used TDIC to analyze temperature and dissolved oxygen time series obtained from automatic measurements in a moored buoy station in coastal waters of Boulogne-Sur-Mer.

The correlation between two data sets is considered here. Suppose the IMFs of two series $x_{1}(t)$ and $x_{2}(t)$ look like $x_{i}(t)=\sum_{j=1}^{N} c_{j}^{i}(t)+r_{i}(t)$ where $c_{j}^{i}(t)$ is the $j^{t h}$ IMF of $x_{i}(t)$ and $r_{i}(t)$ are the residues. We find the instantaneous period $T_{j}^{i}(t)$ of each $c_{j}^{i}(t)$ either by calculating the local extrema points and zero crossing points, i.e.,

$$
T_{j}^{i}(t)=4 \times \frac{\text { data length }}{N b r_{\max }+N b r_{\min }+N b r_{0}} \quad \text { (Huang and Schmitt, }
$$

2014) or by considering the Fourier energy weighted mean frequency, i.e., $T_{j}^{i}(t)=\frac{\int\left|X_{j}^{i}(f)\right|^{2} d f}{\int f\left|X_{j}^{i}(f)\right|^{2} d f}$ where $X_{j}^{i}(f)$ is the Fourier power spectrum of each IMF mode. Then, at time $t_{\text {inst }}$, the sliding window is given by $t_{\text {win }}=\left[t_{\text {inst }}-a \frac{\max \left(T_{j}^{1}(t), T_{j}^{2}(t)\right)}{2}, t_{\text {inst }}+a \frac{\max \left(T_{j}^{1}(t), T_{j}^{2}(t)\right)}{2}\right]$, where $a$ is any positive number between 1 and $\frac{\text { data length }}{2}$. This window is different from classical sliding windows: it is based on the maximum of two instantaneous periods $\max \left(T_{j}^{1}(t), T_{j}^{2}(t)\right)$ and thus it is adaptive. The time-dependent intrinsic correlation of each pair of IMFs is defined as follows: $\operatorname{TDIC}\left(t_{\text {inst }}^{a}\right)=\operatorname{Corr}\left(c_{j}^{1}\left(t_{\text {inst }}^{a}\right), c_{j}^{2}\left(t_{\text {inst }}^{a}\right)\right)$ at any time $t_{\text {inst }}$, where Corr denotes the general correlation coefficient of two time series. Consequently, the TDIC can be interpreted as a time series of correlations between a selected pair of IMFs, determined for each time $t_{\text {inst }}$ using an adaptive window $t_{\text {win }}$, while the variable $a$ has a value from 1 to $\frac{\text { data length }}{2}$.

\section{B. Results for cross analyses: Wavelet coherence results}

The cross correlations between the temperature records were already investigated by Kbaier Ben Ismail et. al., 2015. It was observed that the corresponding time series are highly correlated since the global correlation coefficient is 0.93 for Temp $_{1}$ and Temp 2 with a phase difference of 40 minutes, and 0.86 for Temp $_{1}$ and Temp 4 , occurring when Temp 4 is shifted forwards for about 3 hours. Note that the time lag between stations $S_{1}$ and $S_{2}$ (resp. $S_{4}$ ) corresponds to the propagation speed, which is coherent with the characteristics of these stations in the Réunion island since $S_{2}$ (resp. $S_{4}$ ) is $2.5 \mathrm{~km}$ (resp. $11 \mathrm{~km}$ ) away from $S_{l}$ and the phase velocity is $1 \mathrm{~m} \mathrm{~s}^{-1}$ between them. This result is also observed among the other stations in the island.

Here, cross correlations between $U_{1}$ and $U_{2}$ (the along shore currents time series) are illustrated. The global cross correlation coefficients show a global in-phase relation between the currents time series. Indeed, the time series are highly correlated since the maximum correlation coefficient is 0.72 for $U_{1}$ and $U_{2}$, occurring when $U_{2}$ is shifted forwards for about 10 minutes.

Coherence is defined as the square of the cross spectrum normalized by the individual power spectra. It is a quantity between and that measures the cross correlation between two time series as a function of frequency. The Wavelet Coherence (WTC) finds regions in time frequency space where the two time series co-vary but do not necessarily have high power (Torrence and Webster, 1999; Grinsted et al., 2004). In Fig. 9, we cross the currents spectra from the wavelet decomposition 
method. For $U_{1}$ and $U_{2}$, coherence coefficients are mostly between 0.8 and 0.95 over a wide range of frequencies. As expected, there is a high cross coherence coefficient at times for frequencies approximately $1.39 \mathrm{cpd}$ and $1.93 \mathrm{cpd}$ (periods of 33.48 hours and 12.42 hours, respectively), referring to the inertial wave and the semidiurnal wave frequencies. Since the measurement station for $U_{4}$ is the most distant from the measurement station for $U_{l}$ (see Fig. 1), there is little coherence between the two measures as shown in Fig. 9b). In order to study in detail the cross correlation, the TDIC can be measured for each couple of IMFs.

\section{Time dependent intrinsic correlation results}

The EMD algorithm is first applied to all the data sets for the same time period. The EMD method allows the data sets to be represented in a multiscale way (Flandrin and Gonçalvès, 2004; Huang et al., 1998; Huang, 2009). These are used for multiscale correlation. For example in Fig. 10, we plot the IMFs modes of $U_{1}$ and $U_{2}$ corresponding to the identified semidiurnal tidal wave $\left(I M F_{5}\right)$. These are positively correlated with each other on some portions and negatively correlated on others; showing rich dynamics. The global correlation coefficient between these IMFs is also estimated to 0.55 with a phase difference of 10 minutes, the same as for the original time series $U_{1}$ and $U_{2}$. This result is important since the considered semidiurnal mode is the most energetic one, and it should reflect the global relationship between $U_{1}$ and $U_{2}$. Fig. 11 displays the measured TDIC and shows rich patterns at small sliding window. We note a decorrelation of the TDIC with the increase of the window size. Although the global cross correlation is 0.55 , the TDIC detects several periods of higher/lower correlation between the modes. The decorrelations may highlight the loss of coherency of internal tide over a limited distance of less than $20 \mathrm{~km}$.
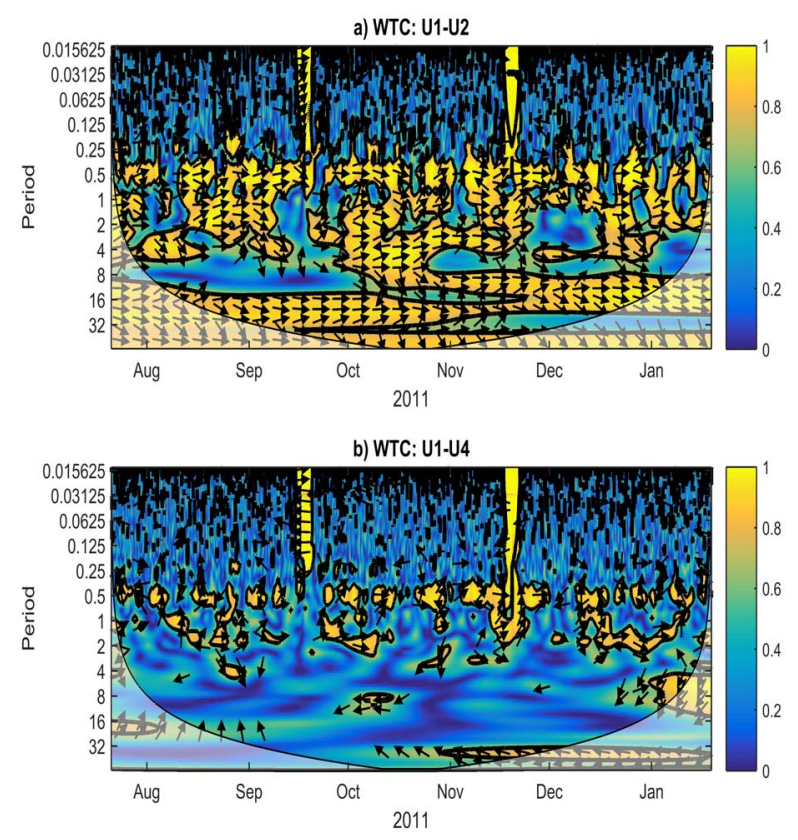

Fig. 9. Crossspectrum between a) $U_{1}$ and $U_{2}$; and b) $U_{1}$ and $U_{4}$ using the wavelet decomposition and coherence measurement. The color-scale represents the value for the coherence from 0 to 1 (blue to yellow). The black arrows represent the phase delay (arrows to the right mean no phase delay).
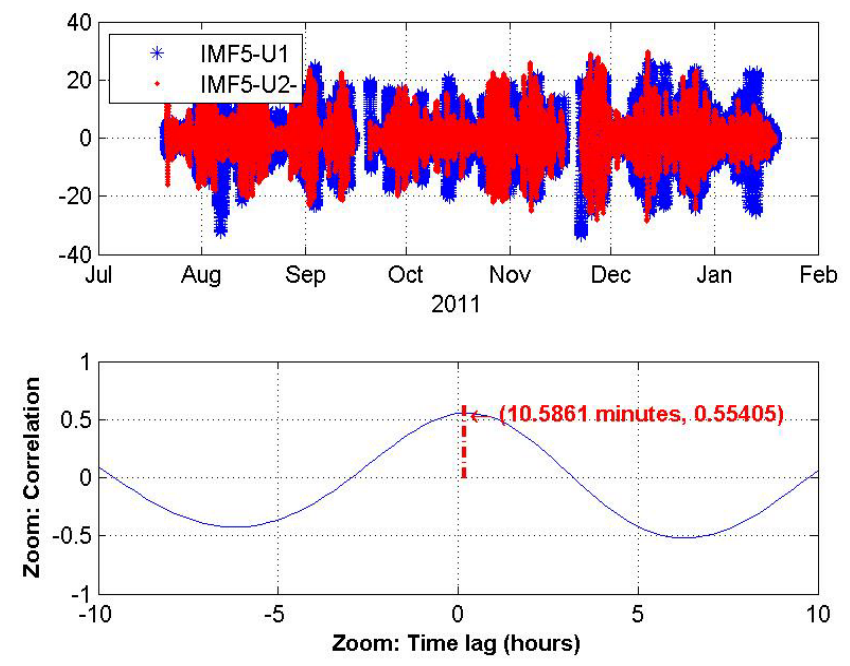

Fig. 10. The semidiurnal cycle from EMD for $U_{1}$ and $U_{2}$ and their correlation.

Moreover, the lower the frequency modes are, the higher the correlation is. To show the time evolution and such scale dependence of cross correlation between the time series, we can consider higher periods such as few days, the weekly cycle, the semimonthly cycle, etc. The residual from EMD algorithm has been recognized as the trend of the given data (Moghtaderi et al., 2011; Wu et al., 2007). With regard to the trends, these are highly correlated: the overall correlation coefficient is nearly 0.86 .

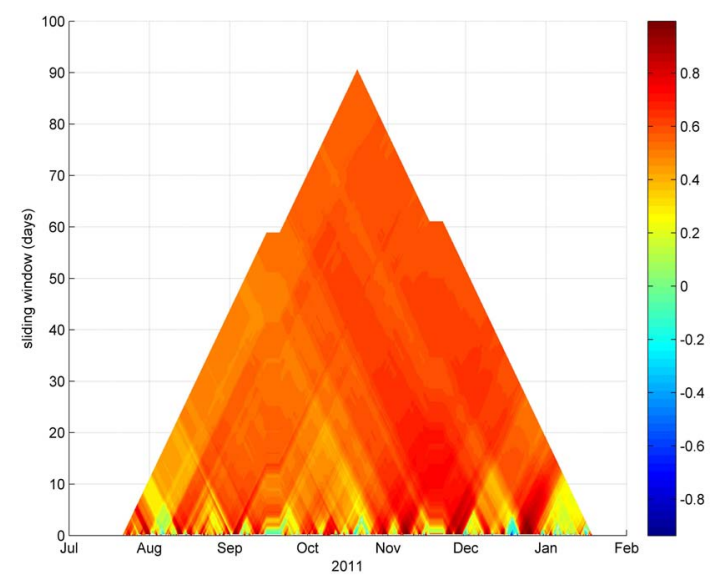

Fig. 11. The measured TDIC between $U_{l}$ and $U_{2}$ for the semidiurnal cycle.

\section{DISCUSSION}

While EMD, Fourier, and wavelets are all used to decompose signals, EMD is fundamentally different from the other two. Some people see the HHT as a generalized Fourier Transform in the sense that the decomposition of the signal of interest by HHT leads to both amplitude and frequency varying time signals. A comparative summary of Fourier, wavelet and HHT analyses is given in Table II. This table 
shows that the HHT is indeed a powerful method for analyzing data from nonlinear and nonstationary processes: it is based on an adaptive basis; the frequency is derived by differentiation rather than convolution; therefore, it is not limited by the uncertainty principle; it is applicable to nonlinear and nonstationary data and presents the results in time-frequency-energy space for feature extraction.

TABLE II. COMAPATIVE SUMMARY OF FOURIER, WAVELET AND HHT

\begin{tabular}{|c|c|c|c|}
\hline \multirow{2}{*}{ Basis } & \multicolumn{3}{|c|}{ Time-frequency techniques } \\
\cline { 2 - 4 } & Fourier & Wavelet & $\boldsymbol{H H T}$ \\
\hline Presentation & $\begin{array}{c}\text { energy- } \\
\text { frequency }\end{array}$ & $\begin{array}{c}\text { energy-time- } \\
\text { frequency }\end{array}$ & $\begin{array}{c}\text { energy-time- } \\
\text { frequency }\end{array}$ \\
\hline Frequency & $\begin{array}{c}\text { convolution: } \\
\text { global } \\
\text { uncertainty }\end{array}$ & $\begin{array}{c}\text { convolution: } \\
\text { regional } \\
\text { uncertainty }\end{array}$ & $\begin{array}{c}\text { differentiation: } \\
\text { local, certainty }\end{array}$ \\
\hline Nonlinear & no & no & yes \\
\hline $\begin{array}{c}\text { Nonstationar } \\
\text { y }\end{array}$ & no & yes & yes \\
\hline $\begin{array}{c}\text { Feature } \\
\text { Extraction }\end{array}$ & no & $\begin{array}{c}\text { discrete: no; } \\
\text { continuous: yes }\end{array}$ & yes \\
\hline $\begin{array}{c}\text { Theoretical } \\
\text { base }\end{array}$ & theory complete & theory complete & empirical \\
\hline
\end{tabular}

The HHT also has disadvantages compared to other spectral analysis methods. The EMD is computationally expensive, especially when the time series is long and has a large frequency distribution. Mode mixing (mixing of different modes in the same scale or separation of the same mode in different scales) appears to be the most significant drawback of EMD, and usually causing intermittency of analyzing signal. Wu and Huang (2009) proposed a noise assisted analysis method, called Ensemble EMD (EEMD), to overcome the mixing problem. The major steps of the EEMD are as follows: First, add white noise to the original time series. Second, create a set of IMFs using EMD. Then, repeat the first two steps with different white noise each time. Finally, take the average of each individual IMF. The result is a new set of IMFs with less mode mixing.

Although HHT and wavelets provide complementary results, HHT is particularly attractive when analyzing signals from complex systems since the EMD decomposes the signal into a finite and small number of Intrinsic Mode Functions (IMFs), which represent different scales of the original time series and physically meaningful modes. Whereas, choosing the wrong basis function (i.e., the wrong mother wavelet) can greatly increase the number of terms required to fit the time series.

\section{CONCLUSION}

Analyzing nonlinear and nonstationary signals remains a very challenging task. The EMD represents a different approach for decomposing such signals, since most methods developed before are based on the concept of Fourier decomposition. Applying the method to temperature records, sea level fluctuations and current data sets in the Réunion island, the inertial wave and many tidal waves have been identified. For the sea level measurements, the two most energetic IMFs refer to the diurnal and semidiurnal waves respectively; and account for over $83 \%$ of the total energy. Whereas for temperature time series, the main part of energy is not carried by the very high frequency components but by intermediate and low ones, especially the seasonal wave. For the currents measurements, the two most energetic IMFs refer to the semidiurnal and the inertial waves respectively; and account for over $48 \%$ of the total energy. Furthermore, the results obtained with HHT approach are compared with CWT approach. In fact, HHT provides superior spatial (temporal) and wavenumber (frequency) resolution for handling nonstationarity and nonlinearity. A noticeable similarity is observed by superimposing IMFs and CWT, although a poor low-frequency resolution is discerned for the Morlet wavelet spectra. Finally, the cross correlation between time series is investigated. While wavelet coherence produces similar results for cross-correlations, it does not provide timefrequency information like TDIC. The time evolution and scale dependencies between two series are considered; and the EMD is used to estimate an adaptive window in order to calculate a TDIC for each pair of IMFs. The trends for temperature and currents time series, representing the residuals from EMD, are highly correlated, whereas higher frequency modes have much smaller correlations. The methodologies presented in this paper are general and thus can be applied on other time series from the environmental and oceanic sciences, where time series are complex with fluctuations over a large range of different spatial and temporal scales, from seconds to thousands of years.

\section{ACKNOWLEDGMENT}

The authors would like to thank the Région Bretagne for financial support of the post-doctoral fellowship (SAD MASTOC). They also thank the Région Réunion for financial support brought to the NortekMed group for the acquisition of the data, provided to IFREMER within the framework of the HydroRun project. Finally, we gratefully acknowledge financial support from the University of Bedfordshire to present the paper at MTS/IEEE Oceans'16.

\section{REFERENCES}

[1] Abarca-Del-Rio, R., Mestre, O., 2006. Decadal to secular time scales variability in temperature measurements over France. Geophys. Res. Lett., 33 (13), L13705, doi: 10.1029/2006GL026019.

[2] Addison, P.S., 2010. The Illustrated Wavelet Transform Handbook: Introductory Theory and Applications in Science, Engineering, Medicine and Finance. Taylor \& Francis, New York.

[3] Avdakovic, S., Ademovic, A., Nuhanovic, A., 2013. Correlation between Air Temperature and Electricity Demand by Linear Regression and Wavelet Coherence Approach: UK, Slovakia and Bosnia and Herzegovina Case Study. Archives of Electrical Engineering, 62 (4), 521-532.

[4] Cazelles, B., Chavez, M., Berteaux, D., Ménard, F., Vik, J., Jenouvrier, S., Stenseth, N.C., 2008. Wavelet analysis of ecological time series. Oecologia 156 (2), 287-304. 
[5] Chen, X., Wu, Z., Huang, N.E., 2010. The time-dependent intrinsic correlation based on the empirical mode decomposition. Adv. Adapt. Data Anal. 2, 233-265.

[6] Dätig, M., Schlurmann, T., 2004. Performance and limitations of the Hilbert-Huang transformation (hht) with an application to irregular water waves. Ocean Eng. 31 (14), 1783-1834.

[7] Ezer, T., Atkinson, L. P., Corlett, W. B., Blanco, J. L., 2013. Gulf Stream's induced sea level rise and variability along the U.S. midAtlantic coast. J. Geophys. Res. Oceans, 118, 685-697.

[8] Flandrin, P., Gonçalvès, P., 2004. Empirical mode decompositions as data-driven wavelet-like expansions. Int. J. Wavelets, Multires. Info. Proc. 2 (4), 477-496.

[9] Flandrin, P., Rilling, G., Gonçalvès, P., 2004. Empirical mode decomposition as a filter bank. IEEE Signal Proc. Lett. 11 (2), 112-114.

[10] Ghorbani, M., 2013. Testing the weak stationarity of a spatio-temporal point process. Stochastic Environmental Research and Risk Assessment 27 (2), 517-524.

[11] Grinsted, A., Moore, J.C., Jevrejeva, S., 2004. Application of the cross wavelet transform and wavelet coherence to geophysical time series. Nonlinear Processes in Geophysics 11 (5/6), 561-566.

[12] Huang, N., 2005. Hilbert-Huang transform and its applications. World Scientific, Ch. 1. Introduction to the Hilbert-Huang Transform and Its Related Mathematical Problems, pp. 1-26.

[13] Huang, N, and Attoh-Okine, N. O., 2005. Hilbert-Huang Transforms in Engineering. CRC Press, Boca Raton, Fla, 313 pp.

[14] Huang, N., Shen S. S. P., 2005. Hilbert-Huang Transform and Its Applications. World Sci., Singapore, $311 \mathrm{pp}$.

[15] Huang, N., Shen, Z., Long, S.R., Wu, M.C., Shih, H.H., Zheng, Q., Yen, N., Tung, C.C., Liu, H.H., 1998. The empirical mode decomposition and the Hilbert spectrum for nonlinear and non-stationary time series analysis. Proc. R. Soc. London, Ser. A 454 (1971), 903-995.

[16] Huang, N., Shen, Z., Long, S.R., 1999. A new view of nonlinear water waves: the Hilbert spectrum. Annu. Rev. Fluid Mech. 31 (1), 417-457.

[17] Huang, N., Wu, Z., 2008. A Review on Hilbert-Huang transform: Method and its Applications to Geophysical Studies, Rev. Geophys., 46 (2), RG2006, doi: 10.1029/2007RG000228.

[18] Huang, Y., 2009. Arbitrary-order Hilbert Spectral Analysis: Definition and Application to Fully Developed Turbulence and Environmental Time Series. (Ph.D. thesis) Université des Sciences et Technologies de Lille — Lille 1, France \& Shanghai University, China.

[19] Huang, Y., Schmitt, F.G., 2014. Time dependent intrinsic correlation analysis of temperature and dissolved oxygen time series using empirical mode decomposition. J. Mar. Syst., 130, 90-100.

[20] Huang, Y., Schmitt, F.G., Hermand, J.-P., Gagne, Y., Lu, Z., Liu, Y., 2011. Arbitrary-order Hilbert spectral analysis for time series possessing scaling statistics: comparison study with detrended fluctuation analysis and wavelet leaders. Phys. Rev. E 84 (1), 016208.

[21] Huang, Y., Schmitt, F., Lu, Z., Liu, Y., 2008. An amplitude-frequency study of turbulent scaling intermittency using Hilbert spectral analysis," Europhys. Lett., vol. 84, 40010.

[22] Jánosi, I., Müller, R., 2005. Empirical mode decomposition and correlation properties of long daily ozone records. Phys. Rev. E 71 (5), 56126.
[23] Kbaier Ben Ismail, D., Lazure, P., Puillat, I., 2015. Advanced spectral analysis and cross correlation based on the empirical mode decomposition: application to the environmental time series, IEEE Geoscience And Remote Sensing Letters, vol. 12 (9), 1968-1972. doi: 10.1109/LGRS.2015.2441374

[24] Leichter, J. J., Stokes, M. D., Hench, J. L., Witting, J. and Washburn, L., 2012. The island scale internal wave climate of Moorea, French Polynesia, J. Geophys. Res. Oceans, vol. 117, 2012, Art. ID. C06008.

[25] Martini, S., Nerini, D., Tamburini, C., 2014. Relation between deep bioluminescence and oceanographic variables: A statistical analysis using time-frequency decompositions. Progress in Oceanography, 127,117-128.

[26] Moghtaderi, A., Borgnat, P., Flandrin, P., 2011. Trend filtering: empirical mode decompositions versus 11 and Hodrick-Prescott. Adv. Adapt. Data Anal. 3 (01n02), 41-61.

[27] Monin, A. S, 1990. Theoretical Geophysical Fluid Dynamics, 399 pp., Kluwer Acad. Publ., Dordrecht.

[28] Pous, S., Lazure, P., Andre, G., Dumas, F., Halo, I., \& Penven, P. (2014). Circulation around La Reunion and Mauritius islands in the south-western Indian Ocean: A modeling perspective. J. Geophys. Res. Oceans, 119(3), 1957-1976. doi:10.1002/2013JC009704

[29] Rao, A.R., Hsu, E.-C., 2008. Hilbert-Huang transform analysis of hydrological and environmental time series, vol. 60. Springer.

[30] Schmitt, F.G., Huang, Y., Lu, Z., Liu, Y., Fernandez, N., 2009. Analysis of velocity fluctuations and their intermittency properties in the surf zone using empirical mode decomposition. J. Mar. Syst. 77, 473-481.

[31] Tatli, H., Dalfes, H. N., Mentes, S. S., 2005. Surface air temperature variability over Turkey and its connection to large-scale upper air circulation via multivariate techniques. Int. J. Climatol., 25, 331-350, doi:10.1002/joc.1133.

[32] Torrence, C., Compo, G., 1998. A practical guide to wavelet analysis. Bulletin of the American Meteorological Society 79 (1), 61-78.

[33] Torrence, C., Webster, P.J., 1999. Interdecadal changes in the ENSOmonsoon system. Journal of Climate 12 (8), 2679-2690.

[34] Wu, S. H., Liu, Z. S., Liu, B. Y., 2006. Enhancement of lidar backscatters signal-to-noise ratio using empirical mode decomposition method. Opt. Commun., 267, 137 -144, doi: 10.1016/ j.optcom.2006.05.069.

[35] Wu, Z., Huang, N.E., 2004. A study of the characteristics of white noise using the empirical mode decomposition method. Proc. R. Soc. Lond. Ser. A 460, 1597-1611.

[36] Wu, Z., and Huang, N.E., 2009. Ensemble empirical mode decomposition: A noise-assisted data analysis method, Adv. Adapt. Data Anal., vol. 1, no. 1, pp. 1-41, 2009.

[37] Wu, Z., Huang, N.E., Long, S.R., Peng, C., 2007. On the trend, detrending, and variability of nonlinear and non-stationary time series. PNAS 104 (38), 14889.

[38] Yin, L., Qiao, F., Zheng, Q., 2014. Coastal-trapped waves in the East China Sea observed by a mooring array in winter 2006. J. Phys. Oceanogr., 44, 576-590. 